\title{
O ensino de semiologia e semiotécnica na ótica de graduandos de enfermagem após o
}

\section{ensino remoto}

The teaching of semiology and semiotechnics from the perspective of nursing graduates after remote teaching

La enseñanza de semiología y semiotecnia desde la perspectiva de los graduados de enfermería después de la enseñanza a distancia

Maria Cidney da Silva Soares

ORCID: https://orcid.org/0000-0002-8150-108X Centro Universitário Unifacisa, Brasil E-mail: cidney.soares@unifacisa.edu.br

Ana Beatriz do Nascimento Silva Amaral ORCID: https://orcid.org/0000-0002-8451-6041 Centro Universitário Unifacisa, Brasil

E-mail: ana.amaral@maisunifacisa.com.br

Clara Stefhanie Medeiros do Nascimento ORCID: https://orcid.org/0000-0003-4479-1425 Centro Universitário Unifacisa, Brasil

E-mail:clara.nascimento@maisunifacisa.com.br

Danielle Cavalcante de Farias

ORCID: https://orcid.org/0000-0001-5054-504X Centro Universitário Unifacisa, Brasil

E-mail: danielle.farias@maisunifacisa.com.br

Hellen Dayanna da Paz Silva

ORCID: https://orcid.org/0000-0002-2012-1323 Centro Universitário Unifacisa, Brasil

E-mail: hellen.dayanna@maisunifacisa.com.br

Laryssa Portela de Araújo

ORCID: https://orcid.org/0000-0003-0312-8627 Centro Universitário Unifacisa, Brasil

E-mail: laryssa.araujo@maisunifacisa.com.br

Maria Eduarda Araujo Ribeiro

ORCID: https://orcid.org/0000-0002-1245-649X Centro Universitário Unifacisa, Brasil

E-mail: maria.eduarda.ribeiro@maisunifacisa.com.br Maria Vitória Alves Dantas

ORCID: https://orcid.org/0000-0003-1936-8879 Centro Universitário Unifacisa, Brasil

E-mail: maria.dantas@maisunifacisa.com.br

Maria Eduarda Soares Marinho

ORCID: https://orcid.org/0000-0002-3695-8507 Centro Universitário Unifacisa, Brasil

E-mail: maria.eduarda@maisunifacisa.com.br

Patrícia Tayse de Lima Soares

ORCID: https://orcid.org/0000-0003-4414-1078 Centro Universitário Unifacisa, Brasil

E-mail:patricia.soares@maisunifacisa.com.br

Wellen Cristina da Silva Dantas ORCID: https://orcid.org/0000-0003-2809-7716 Centro Universitário Unifacisa, Brasil

E-mail:wellen.dantas@maisunifacisa.com.br

\section{Resumo}

Identificar o entendimento e satisfação de graduandos de enfermagem que estão cursando ou cursaram disciplinas práticas, incluindo semiologia e semiotécnica de forma remota. Estudo descritivo, exploratório, quantitativo, desenvolvido via online após autorização do comitê de ética e pesquisa. Amostra composta por mulheres $(47,1 \% ; n=32)$ entre 18 e 29 anos $(44,1 \%)$ da região nordeste $(54,4 \% ; n=37)$, morando com familiares $(57,4 \% ; n=39)$, renda entre um e três salários mínimos $(44,1 \%$; $=30)$, trabalhando $(38,2 \% ; n=26)$ em tecnologia $(20,6 \% ; n=14)$ de instituição privada 
$(61,8 \% ; n=42)$, de manhã $(33,8 \% ; n=23)$ quarto período $(23,5 \% ; n=16)$. Acesso à internet $(61,8 \% ; n=42)$ com financiamento $(41,6 \% ; n=31)$ não possuíam outro curso superior $(55,9 \% ; n=38)$ ou curso técnico $(33,8 \% ; n=23)$. Embora as instituições desses indivíduos tenham aderido ao ensino remoto das aulas práticas $(55,9 \%$; $\mathrm{n}=38)$, estes assistiram as aulas de semiologia e semiotécnica no formato presencial $(33,8 \% ; n=23)$. As maiores médias de satisfação dos discentes estiveram presentes nas questões de "infraestrutura física da instituição", "das salas de aula" e "relacionamento com os professores". Por sua vez, as menores médias foram relativas à "Assistir aulas práticas em casa de forma remota da disciplina semiologia e semiotécnica", "mudar do ensino presencial para o ensino remoto nas disciplinas práticas incluindo semiologia e semiotécnica" e "preparação para executar procedimentos técnicos de semiologia e semiotécnica após as aulas remotas". Conclui-se que é necessário uma conciliação entre a educação digital e a formação de profissionais na área de saúde e que alunos de enfermagem preferem aulas práticas presenciais na disciplina de semiologia e semiotécnica.

Palavras-chave: Enfermagem; Ensino híbrido; COVID-19; Ensino; Semiologia e semiotécnica.

\begin{abstract}
Identify the understanding and satisfaction of nursing undergraduates who are taking or have attended practical subjects, including semiology and semiotechnics remotely. Descriptive, exploratory, quantitative study, developed online after authorization from the research ethics committee. Sample composed of women $(47.1 \%$; $n=32)$ between 18 and 29 years $(44.1 \%)$ from the northeast region $(54.4 \% ; n=37)$, living with family members $(57.4 \% ; n=39)$, income between one and three minimum wages $(44.1 \% ; n=30)$, working $(38.2 \% ; n=26)$ in technology $(20.6 \% ; n=14)$ in a private institution $(61,8 \% ; n=42)$, in the morning $(33.8 \% ; n=23)$ fourth period $(23.5 \% ; n=16)$. Internet access $(61.8 \% ; n=42)$ with financing $(41.6 \% ; n=31)$ did not have another higher education course $(55.9 \% ; n=38)$ or technical course $(33.8 \% ; n$ =23). Although the institutions of these individuals have adhered to remote teaching of practical classes $(55.9 \%$; $n=38)$, they attended semiology and semiotechnics classes in face-to-face format $(33.8 \% ; n=23)$. The highest averages of student satisfaction were present in the questions of "institution's physical infrastructure", "classrooms" and "relationship with teachers". In turn, the lowest averages were related to "Watching practical classes at home remotely in the semiology and semiotechnics subject", "switching from face-to-face teaching to remote teaching in practical subjects including semiology and semiotechnics" and "preparation to perform technical procedures of semiology and semiotechnics after remote classes". It is concluded that a reconciliation between digital education and the training of professionals in the health area is necessary, and that nursing students prefer face-to-face practical classes in the discipline of semiology and semiotechnics.
\end{abstract}

Keywords: Nursing; Hybrid teaching; COVID-19; Teaching; Semiology and semiotechnics.

\title{
Resumen
}

Identificar la comprensión y satisfacción de los estudiantes de enfermería que cursan o han cursado asignaturas prácticas, incluidas semiología y semiotécnica de forma remota. Estudio descriptivo, exploratorio, cuantitativo, desarrollado online previa autorización del comité de ética en investigación. Muestra compuesta por mujeres $(47,1 \% ; n=32)$ entre 18 y 29 años $(44,1 \%)$ de la región noreste $(54,4 \% ; n=37)$, que viven con familiares $(57,4 \% ; n=39)$, ingresos entre uno y tres salarios mínimos $(44,1 \% ; n=30)$, trabajando $(38,2 \% ; n=26)$ en tecnología $(20,6 \% ; n=14)$ en una institución privada $(61,8 \% ; n=42)$, por la mañana $(33,8 \% ; n=23)$ cuarto período $(23,5 \% ; n=16)$. El acceso a Internet $(61,8 \% ; n$ $=42)$ con financiamiento $(41,6 \% ; n=31)$ no tenía otro curso de educación superior $(55,9 \% ; n=38)$ ni curso técnico $(33,8 \% ; n=23)$. Si bien las instituciones de estos individuos se han adherido a la enseñanza a distancia de clases prácticas $(55,9 \% ; n=38)$, asistieron a clases de semiología y semotecnología en formato presencial $(33,8 \% ; n=23)$. Los mayores promedios de satisfacción de los estudiantes se presentaron en las preguntas de "infraestructura física de la institución", "aulas" y "relación con los docentes". A su vez, los promedios más bajos se relacionaron con "Asistir a clases prácticas en casa de forma remota en la asignatura de semiología y semiotécnica", "pasar de la enseñanza presencial a la enseñanza a distancia en asignaturas prácticas que incluyen semiología y semiotécnica" y "preparación para realizar prácticas técnicas". procedimientos de semiología y semiotecnia después de clases a distancia". Se concluye que es necesaria una conciliación entre la educación digital y la formación de profesionales en el área de la salud, y que los estudiantes de enfermería prefieren las clases prácticas presenciales en la disciplina de semiología y semiotecnología.

Palabras clave: Enfermería; Enseñanza híbrida; COVID-19; Enseñanza; Semiología y semiotecnia.

\section{Introdução}

A disciplina de semiologia e semiotécnica é a base assistencial para os cursos de graduação em enfermagem, sendo, portanto, necessária à construção de conhecimentos que possibilitem a efetivação do cuidado nos múltiplos ambientes em que o profissional de enfermagem está inserido. O ensino dessa disciplina consolida-se por meio de atividades teórico-práticas, 
fundamentais para desfechar a reflexão crítica, agenciar a apreensão de conceitos-base e o desenvolvimento de habilidades e competências com base em situações simuladas ou reais de cuidado (Melo et. al, 2017).

Segundo Porto (2017) e Barros (2016) para aprender semiologia e semiotécnica o essencial continua sendo o contato direto com os pacientes, sendo evidenciado que é a única maneira de se alcançar o verdadeiro aprendizado das profissões da saúde. No entanto, com o advento da pandemia que assolou a humanidade a partir do ano de 2019, causada pela Covid 19, a educação no ensino superior precisou de forma repentina tomar outros rumos.

Sabendo que as disciplinas práticas são de suma importância para formação do enfermeiro, essa nova realidade tem levantado discussões em relação a formação técnica científica e crítica de novos enfermeiros, analisando o estudo realizado por Souza et. al 2020, fica constatado que o aluno que nas aulas de semiologia e semiotécnica que utilizam de simulação realística tem maior autoconfiança e satisfação na sua formação, e isto tem deixado a comunidade docente preocupada, pois, diante desse novo contexto educacional em virtude da paralisação de aulas laboratoriais e em serviços de saúde.

Em decorrência da pandemia, as aulas práticas têm sido substituídas por aulas remotas, o que foi autorizado pela Portaria do MEC n 544 de 16 de junho de 2020, que dispõe sobre aulas em meio digitais enquanto durar a pandemia do Novo Coronavírus (COVID-19), e ainda, estabelece o ensino remoto emergencial com o objetivo de garantir aos discentes a entrega dos conteúdos programados estendendo tais recomendações às aulas práticas, incluindo os estágios curriculares supervisionados, podendo estes serem realizados de forma presencial e/ou remota. (Brasil, 2020).

Entendendo que se faz necessário a reflexão crítica do graduando em Enfermagem acerca da importância dessa disciplina como instrumento metodológico que incrementa a prática acadêmica e ainda oferece subsídios para o exercício profissional, esse estudo lança a seguinte questão norteadora: Qual o entendimento de graduandos de enfermagem que estão cursando ou já cursaram a disciplina de semiologia e semiotécnica de forma remota sobre o ensino aprendizagem dela?

Dessa forma, construiu-se os seguintes objetivos: Identificar o entendimento e satisfação de graduandos de enfermagem que estão cursando ou cursaram disciplinas práticas, incluindo semiologia e semiotécnica de forma remota. E como objetivos específicos: Avaliar o nível de satisfação dos discentes de acordo com a escala Escala de Satisfação com a Experiência Acadêmica (ESEA); Correlacionar o nível e as dimensões de satisfação da ESEA com as demais variáveis da pesquisa; Compreender a experiência discente com o ensino remoto de Semiologia e Semiotécnica; Triangular os dados da compreensão discente e resultados da ESEA.

\section{Metodologia}

Trata-se de uma pesquisa de caráter exploratória, descritiva com abordagem quantitativa.

A pesquisa exploratória possibilita a descoberta, o esclarecimento de fenômenos ou a explicação daqueles que não eram aceitos apesar de evidentes (Gonçalves, 2014). A pesquisa descritiva tem por objetivo levantar as opiniões, atitudes e crenças de uma população, bem como encontrar pontos em comum entre as variáveis em questão. No entanto, algumas pesquisas definidas como descritivas, a partir de seus objetivos, acabam proporcionando uma nova visão do problema, o que as aproxima das pesquisas exploratórias (Gil, 1999).

Quanto ao método quantitativo é utilizado no desenvolvimento de pesquisas descritivas, auxiliando na descoberta e classificação em relação às variáveis, assim como na investigação de causalidade entre os fenômenos: causa e efeito. Visa, a garantia e precisão dos resultados evitando desvios na análise e interpretação (Oliveira, 1997).

A pesquisa em questão pode ser classificada como um levantamento de campo (survey), de acordo com Gil (1999), este tipo de pesquisa se caracteriza pelo questionamento direto das pessoas com a finalidade de conhecer os comportamentos desejados. Nela, a solicitação de informações é feita diretamente a um grupo significativo de pessoas acerca do problema 
estudado e, através de análise quantitativa, atinge as conclusões correspondentes dos dados coletados. Desta forma, os métodos citados acima formam uma boa composição para esta pesquisa por se tratar de uma problemática muito relevante, e que diante do contexto pandêmico, mostrou-se ainda mais relevante diante das demandas do perfil estudantil, bem como do mercado de trabalho que irá receber profissionais qualificados de acordo com o que lhes foi oferecido.

A pesquisa foi realizada em meio virtual com graduandos de enfermagem de qualquer local do país que estavam cursando ou cursaram a disciplina de semiologia e semiotécnica em enfermagem durante a pandemia da COVID 19.

A população foi composta por todos os graduandos de enfermagem de todo país e a amostra foi do tipo probabilística, aleatória simples, por definição de população infinita. Foram incluídos alunos de enfermagem matriculados na disciplina de semiologia e semiotécnica no momento da coleta dos dados e alunos do curso de enfermagem que cursaram a disciplina no período da pandemia COVID 19 e tiveram as aulas de semiologia e semiotécnica de forma remota. Sendo excluídos alunos que não estão cursando ou ainda não cursaram a disciplina de semiologia e semiotécnica e alunos que cursaram mas não são do curso de enfermagem.

O formulário foi construído e disponibilizado em endereço eletrônico, onde as pesquisadoras tiveram acesso às respostas na medida em que os entrevistados as enviavam, recebendo em tempo real a notificação das respostas através de e-mail.

A ferramenta Google Forms, é um formulário eletrônico que possui inúmeras vantagens como: a possibilidade de acesso em qualquer local ou horário; a economia de espaço no disco rígido; o fato de ser gratuito; a facilidade de uso, pois não requer conhecimentos de programação; e uma interface amigável (Mathias \& Sakai, 2013).

A ferramenta foi condicionada em suas configurações a aceitar somente uma resposta por cada $e$-mail, evitando assim, a duplicidade de dados nas respostas. Na primeira parte da pesquisa era obrigatório um endereço de $e$-mail válido, o qual recebia de forma automática uma cópia do formulário preenchido, posteriormente os candidatos passaram por um filtro e só seria disponibilizado o TCLE para aqueles que assinalaram a opção "Sou aluno(a) do curso de enfermagem em instituição pública ou privada". Aos que marcarem a opção "Não sou aluno(a) do curso de enfermagem” o formulário foi encerrado. Após este filtro, tinha outro que identificava se o aluno tinha cursado ou não a disciplina de semiologia e semiotécnica de forma remota para só assim prosseguir como respondente da pesquisa.

Seguindo o Ofício Circular No 2/2021/CONEP/SECNS/MS, publicado em 24 de fevereiro de 2021, que traz orientações para procedimentos em pesquisas com qualquer etapa em ambiente virtual, o convite para participação com o link da pesquisa foi enviado de forma individual e cada $e$-mail e/ou mensagem por aplicativos de comunicação (WhatsApp) composto apenas por um remetente e um destinatário, ou enviados na forma de lista oculta. De forma a não permitir a identificação dos convidados nem a visualização dos seus dados de contato (e-mail, telefone, etc.) por terceiros.

No mesmo convite foi esclarecido aos participantes de pesquisa, que antes de responder às perguntas disponibilizadas em ambiente não presencial ou virtual, seria apresentado o Termo de Consentimento Livre e Esclarecido (TCLE) para a sua anuência. Também foi enfatizado a importância dos participantes da pesquisa, guardar em seus arquivos uma cópia do documento eletrônico pois, ao final da pesquisa, foi enviado de forma automática, para todos os participantes uma cópia do formulário com o TCLE assinado de forma digital. Durante o período da coleta de dados, foi disponibilizado um contato para que, via e-mail, os participantes pudessem encaminhar suas possíveis dúvidas e receber os devidos esclarecimentos.

Após a coleta foi feito download dos dados coletados para um dispositivo eletrônico local, apagando todo e qualquer registro de qualquer plataforma virtual, ambiente compartilhado ou "nuvem".

Os dados foram exportados do Microsoft Excel® 2013 para análise no software estatístico Statistical Package for the Social Sciences SPSS, versão 26.0, por meio de estatística descritiva (frequências absoluta e relativa, medidas de tendência central e dispersão). 
Foi seguida a RESOLUÇÃO n 466/2012 do Conselho Nacional de Saúde do Ministério da Saúde (BRASIL, 2012) que dispõe das diretrizes que regulamenta a pesquisa com seres humanos só sendo operacionalizado a partir da anuência deste órgão sob CAAE: 45787721.8 .0000 .5175 .

\section{Resultados e Discussão}

A Tabela 1 expressa o perfil sociodemográfico dos participantes, em que a maioria foi composta por mulheres (47,1\%; $\mathrm{n}=32)$, com idade entre 18 e 29 anos $(44,1 \%$; $=30)$, naturais da região nordeste $(54,4 \% ; n=37)$, morando com familiares $(57,4 \%$; $\mathrm{n}=39)$, renda entre um e três salários mínimos $(44,1 \% ; n=30)$, e trabalhando $(38,2 \% ; n=26)$ na área de tecnologia $(20,6 \%$; $=14)$. Sublinha-se que nenhum dos entrevistados era portador de necessidades especiais.

Tabela 1 - Distribuição das variáveis sociodemográficas dos participantes. Campina Grande, PB, Brasil, 2021. (N=68)

\begin{tabular}{|c|c|c|}
\hline Variáveis & $\mathbf{N}(\%)$ & Amostra em falta \\
\hline \multicolumn{3}{|l|}{ Sexo } \\
\hline Feminino & $32(47,1)$ & \multirow{2}{*}{$42 / 26$} \\
\hline Masculino & $10(14,7)$ & \\
\hline \multicolumn{3}{|l|}{ Faixa etária } \\
\hline 18 aos 29 anos & $30(44,1)$ & \multirow{2}{*}{$42 / 26$} \\
\hline 30 aos 49 anos & $12(17,6)$ & \\
\hline \multicolumn{3}{|l|}{ Região de naturalidade } \\
\hline Nordeste & $37(54,4)$ & \multirow{4}{*}{$42 / 26$} \\
\hline Norte & $1(1,5)$ & \\
\hline Sudeste & $3(4,4)$ & \\
\hline Sul & $1(1,5)$ & \\
\hline \multicolumn{3}{|l|}{ Arranjo de moradia } \\
\hline Sozinho (a) & $1(1,5)$ & \multirow{3}{*}{$42 / 26$} \\
\hline Familiares & $39(57,4)$ & \\
\hline Outros & $2(2,9)$ & \\
\hline \multicolumn{3}{|l|}{ Renda } \\
\hline Menos de 1 salário mínimo & $6(8,82)$ & \multirow{3}{*}{$42 / 26$} \\
\hline Entre 1 e 3 salários mínimos & $30(44,1)$ & \\
\hline Mais de 3 salários mínimos & $6(8,82)$ & \\
\hline \multicolumn{3}{|c|}{ Portador de necessidade especial } \\
\hline Sim & $0(0,0)$ & \multirow{2}{*}{$42 / 26$} \\
\hline Não & $42(61,8)$ & \\
\hline \multicolumn{3}{|l|}{ Trabalha } \\
\hline Sim & $26(38,2)$ & \multirow{2}{*}{$42 / 26$} \\
\hline Não & $16(23,5)$ & \\
\hline \multicolumn{3}{|l|}{ Se sim, em qual área? } \\
\hline Área da saúde & $12(17,6)$ & \multirow{2}{*}{$42 / 26$} \\
\hline Área de tecnologia & $14(20,6)$ & \\
\hline
\end{tabular}

Fonte: Autores (2021).

De acordo com os achados da pesquisa, evidenciou-se que mulheres jovens procuram mais o ensino superior do que os homens, isso porque, segundo Barreto (2012), a universidade e a pesquisa científica exercem considerável importância para o papel educacional e político auxiliando o desenvolvimento de projetos brasileiros, servindo não só como instrumento de emancipação feminina e masculina por meio da formação profissional, como também por ofertar à sociedade uma reflexão crítica sobre si mesma. Por isso, Ferreira et al. (2011) também explica esse empoderamento feminino na sociedade, já que, com as 
guerras mundiais, a mão de obra masculina ficou escassa, necessitando, então, do ingresso das mulheres no mercado de trabalho, mesmo com salários baixos. Com o tempo, a presença feminina ganhou espaço, destacando-se em diversos setores da economia.

No que diz respeito à inserção de estudantes com deficiência no ensino superior, constatou-se que não há um lugar de destaque, principalmente no curso de enfermagem. Na década de 1990, houve mudanças de enorme significado para as ações de políticas públicas educacionais, principalmente no que diz respeito à escolarização de alunos com deficiência, o que proporcionou, então, avanços inimagináveis para a efetivação de uma Escola para todos, o que configura um princípio fundamental da inclusão (Garcia et al., 2018).

No entanto, ao longo de processos históricos, a discriminação, exclusão social e desrespeito aos direitos das pessoas com deficiência, são fatos que perpetuam desde a antiguidade. Com isso, essas pessoas acabam sofrendo condutas que afrontam seus direitos e dificultam o exercício da cidadania, inerentes à condição humana (Wellichan \& Souza, 2017).

Somado a isso, tem-se também outra realidade entre os acadêmicos de enfermagem: trabalhar para estudar. A acelerada expansão do ensino superior no país trouxe para a população a necessidade cada vez maior de se fazer um curso superior para poder ter uma estabilidade financeira futura. Pois, é notório que, aqueles que não estudam, acabam sentindo mais dificuldades de serem inseridos no mercado de trabalho do que outros que já tem ensino superior (Abrantes, 2012).

Segundo o mesmo autor, existe um grupo de pessoas de menor renda que são ainda mais prejudicados com essa cobrança. Porque, geralmente, precisam frequentar cursos noturnos e trabalhar durante o dia para poder arcar com as despesas tanto pessoais quanto do próprio curso. Essas pessoas sofrem por não poder abandonar seus trabalhos e dedicar-se aos estudos, pois grande parte desse público depende dele para dar continuidade ao curso. Por isso, percebe-se que existem inúmeras dificuldades para serem enfrentadas, pois essa dupla jornada é exaustiva e requer, muitas vezes, a abdicação do lazer para dar conta da demanda tanto do trabalho quanto dos estudos.

Com relação aos aspectos acadêmicos nesse estudo, verificou-se a prevalência de estudantes vinculados à instituição privada $(61,8 \% ; n=42)$, matriculados na turma da manhã $(33,8 \% ; n=23)$ e no quarto período $(23,5 \%$; $=16)$. Ademais, tinham acesso à internet e aparelhos digitais $(61,8 \% ; n=42)$, recebiam subsídios de financiamento $(41,6 \%$; n=31) e não possuíam outro curso superior $(55,9 \% ; n=38)$ ou curso técnico $(33,8 \% ; n=23)$. Embora as instituições desses indivíduos tenham aderido ao ensino remoto das aulas práticas $(55,9 \%$; $\mathrm{n}=38)$, estes assistiram as aulas de semiologia e semiotécnica no formato presencial $(33,8 \%$; $\mathrm{n}=23$ ) (Tabela 2). 
Tabela 2 - Características acadêmicas dos participantes. Campina Grande, PB, Brasil, 2021. (N=68)

\begin{tabular}{|c|c|c|}
\hline Variáveis & $\mathbf{N}(\%)$ & Amostra em falta \\
\hline \multicolumn{3}{|c|}{ Tipo de Instituição de Ensino Superior } \\
\hline Privada & $42(61,8)$ & \multirow{2}{*}{$42 / 26$} \\
\hline Pública & $0(0,0)$ & \\
\hline \multicolumn{3}{|c|}{ Turno em que estuda } \\
\hline Manhã & $23(33,8)$ & \multirow{3}{*}{$42 / 26$} \\
\hline Noite & $11(16,2)$ & \\
\hline Integral & $7(10,3)$ & \\
\hline \multicolumn{3}{|c|}{ Vínculo no semestre } \\
\hline $3^{\circ}$ & $8(11,8)$ & \multirow{6}{*}{$42 / 26$} \\
\hline $4^{\circ}$ & $16(23,5)$ & \\
\hline $5^{\circ}$ & $13(19,1)$ & \\
\hline $6^{\circ}$ & $4(4,4)$ & \\
\hline $7^{\circ}$ & $1(1,5)$ & \\
\hline $9^{\circ}$ & $1(1,5)$ & \\
\hline \multicolumn{3}{|c|}{ Tem acesso à internet em casa } \\
\hline Sim & $42(61,8)$ & \multirow{2}{*}{$42 / 26$} \\
\hline Não & $0(0,0)$ & \\
\hline \multicolumn{3}{|c|}{$\begin{array}{l}\text { Tem acesso à computador, tablets ou smartphone em } \\
\text { casa }\end{array}$} \\
\hline Sim & $42(61,8)$ & \multirow{2}{*}{$42 / 26$} \\
\hline Não & $0(0,0)$ & \\
\hline \multicolumn{3}{|c|}{$\begin{array}{l}\text { Recebe algum tipo de subsídio para financiamento do } \\
\text { curso }\end{array}$} \\
\hline Sim & $31(45,6)$ & \multirow{2}{*}{$42 / 26$} \\
\hline Não & $11(16,2)$ & \\
\hline \multicolumn{3}{|c|}{ Possui outro curso superior } \\
\hline Sim & $4(5,9)$ & \multirow{2}{*}{$42 / 26$} \\
\hline Não & $38(55,9)$ & \\
\hline \multicolumn{3}{|c|}{ Possui curso técnico } \\
\hline Sim & $19(27,9)$ & \multirow{2}{*}{$42 / 26$} \\
\hline Não & $23(33,8)$ & \\
\hline \multicolumn{3}{|c|}{$\begin{array}{l}\text { A sua instituição aderiu ao ensino de aulas práticas } \\
\text { de forma remota? }\end{array}$} \\
\hline Sim & $36(52,9)$ & \multirow{2}{*}{$42 / 26$} \\
\hline Não & $6(8,8)$ & \\
\hline \multicolumn{3}{|c|}{$\begin{array}{l}\text { Como você assiste/assistiu as aulas práticas de } \\
\text { semiologia e semiotécnica? }\end{array}$} \\
\hline Presencial & $23(33,8)$ & \multirow{2}{*}{$42 / 26$} \\
\hline Remoto & $19(27,9)$ & \\
\hline
\end{tabular}

Fonte: Autores.

O uso de tecnologia no século XXI é uma realidade no Brasil, porém, antigamente a geração Baby Boom (crianças nascidas durante uma explosão populacional), na década de 70, não tinha acesso a tecnologia como nos dias atuais precisavam de pessoas que já dispusessem de conhecimento para se aprofundar nos estudos, de outro lado temos uma geração considerada filhos da tecnologia nascidos na década de 80, os quais se adaptam e têm acesso mais facilmente a novas tecnologias inseridas principalmente no âmbito da educação.

Ademais essas gerações entram em conflito, considerando que modelos padronizados de educação com professor, quadro, carteiras, salas de aula, e normalmente, sem metodologia ativa, são encarados como meios de ensino monótonos pelos "filhos da tecnologia", entende-se portanto, que é necessário considerar o uso da tecnologia como alternativa para melhoria no ensino e aprendizagem dos alunos, formando seres críticos (Segantini, 2014).

Em relação aos dados encontrados na pesquisa, constatou-se uma necessidade de aderir ao ensino remoto. Isso porque, com o advento da pandemia, causada pelo SARS- COV-2, foi necessário adotar medidas para controle, como a suspensão das atividades acadêmicas presenciais com objetivo de conter o avanço da pandemia. Com isso, foram criadas portarias que possibilitaram o desenvolvimento de metodologias remotas, entretanto, não foi levado em consideração o ensino de práticas e 
estágio na formação em saúde que são fundamentais para a formação do enfermeiro. Nesta nova modalidade, o corpo docente pode elaborar um planejamento didático usando ferramentas no Google como Meet, Classroom, Docs, Planilhas, Apresentações, Agenda, Jamboard entre outros visando a interação e inovação (Spagnol et al.,2020; Fernandes et al., 2021; Camacho, 2020).

A prática de Educação a distância $(\mathrm{EaD})$ tem sido evidenciada como uma ferramenta capaz de gerar um aprendizado contínuo, a qual reconhece a coletividade, a cooperação e a interação como marcadores de um processo de ensino-aprendizagem, além de possibilitar a integração de mídias para simulações de cenários de práticas e a experimentação do aluno nessas aulas sem que haja risco para a vida de um paciente real. Todavia, existem alguns pontos negativos que cercam essa nova modalidade de ensino, sendo elas as mudanças de rotina, principalmente em relação ao contexto do distanciamento social e na divisão de equipamentos e ambientes com outros membros, como também prejuízos na formação dos profissionais da área de saúde, devido ao risco que eles possam causar à sociedade (Comin et al., 2020; Lira et al., 2020).

Tendo em vista o caráter emergencial que se apresenta a atual situação do Brasil, o ensino a distância tem ganhado espaço sendo necessário desenvolver medidas inovadoras no processo de ensino e aprendizagem, entretanto é notório a desvalorização do estudante em relação ao rendimento do ensino remoto evidenciando a urgência do ensino presencial pautado na interação humana ( Rigiel et al., 2021; Soares et al., 2021). Mesmo antes da pandemia, o ensino de práticas de enfermagem à distância já se mostrava uma pauta relevante, levando-se em consideração, seus impactos para a sociedade. O Ministério Público Federal, por meio do Ofício LLO/PRDF/MPF nº 2.896/2015, solicitou posicionamento oficial do Conselho Federal de Enfermagem (COFEN) quanto à situação do ensino EaD em enfermagem no contexto nacional, que concluiu que a assistência à saúde da população poderia está em risco significativo, de acordo com os resultados da chamada "Operação EaD”, realizada em resposta ao ofício referido que resultou no Relatório Consubstanciado da Operação EaD (COFEN, 2016).

Foi possível identificar que as maiores médias de satisfação dos discentes estiveram presentes nas questões de "infraestrutura física da instituição", "infraestrutura física das salas de aula" e "relacionamento com os professores". Por sua vez, as menores médias foram relativas à "experiência assistindo aulas práticas em casa de forma remota da disciplina semiologia e semiotécnica" "experiência ao mudar do ensino presencial para o ensino remoto nas disciplinas práticas incluindo semiologia e semiotécnica" e "preparação para executar procedimentos técnicos de semiologia e semiotécnica após as aulas remotas" (Tabela $3)$. 
Tabela 3 - Média de satisfação dos discentes de acordo com alguns itens da Escala de Satisfação com a Experiência Acadêmica e o ensino remoto de Semiologia e Semiotécnica. Campina Grande, PB, Brasil, 2021. (N=68)

\begin{tabular}{|c|c|c|}
\hline $\mathbf{N}^{\mathbf{0}}$ & Itens do questionário & Média \pm DP \\
\hline 01 & Experiência nas aulas práticas (de forma geral) durante o período de pandemia & $2,00 \pm 1,01$ \\
\hline 02 & $\begin{array}{l}\text { Experiência assistindo aulas práticas em casa de forma remota da disciplina semiologia } \\
\text { e semiotécnica }\end{array}$ & $1,79 \pm 0,92$ \\
\hline 03 & $\begin{array}{l}\text { Experiência assistindo aulas práticas na faculdade da disciplina semiologia e } \\
\text { semiotécnica }\end{array}$ & $2,83 \pm 1,17$ \\
\hline 04 & Relacionamento com os professores & $3,67 \pm 1,07$ \\
\hline 05 & Diversidade das atividades extracurriculares oferecidas pela instituição & $2,83 \pm 1,19$ \\
\hline 06 & Eventos sociais oferecidos pela instituição & $2,91 \pm 1,23$ \\
\hline 07 & Envolvimento pessoal nas atividades do curso & $2,95 \pm 0,99$ \\
\hline 08 & Programas ou serviços de apoio aos estudantes oferecidos pela instituição & $2,62 \pm 1,32$ \\
\hline 09 & Condições oferecidas para o meu desenvolvimento profissional & $2,50 \pm 1,17$ \\
\hline 10 & Condições para ingresso na área profissional de formação & $2,50 \pm 1,15$ \\
\hline 11 & Programa de apoio financeiro oferecido pela instituição & $2,81 \pm 1,33$ \\
\hline 12 & Oportunidade de desenvolvimento pessoal oferecida pela instituição & $2,71 \pm 1,27$ \\
\hline 13 & $\begin{array}{l}\text { Adequação entre o meu investimento financeiro para custear os estudos e a formação } \\
\text { recebida }\end{array}$ & $2,64 \pm 1,25$ \\
\hline 14 & Recursos e equipamentos audiovisuais disponíveis na instituição & $3,12 \pm 1,21$ \\
\hline 15 & Atendimento e clareza das informações oferecidas pelos funcionários da secretaria & $3,17 \pm 1,34$ \\
\hline 16 & Equipamentos e softwares oferecidos pelo laboratório de informática & $3,14 \pm 1,20$ \\
\hline 17 & Atendimento e clareza das informações oferecidas pelos funcionários da biblioteca & $3,31 \pm 1,24$ \\
\hline 18 & Acervo disponível na biblioteca & $3,43 \pm 1,15$ \\
\hline 19 & Segurança oferecida pela instituição & $3,41 \pm 1,13$ \\
\hline 20 & Infraestrutura física das salas de aula & $3,81 \pm 1,13$ \\
\hline 21 & Infraestrutura física da instituição & $3,95 \pm 1,03$ \\
\hline 22 & $\begin{array}{l}\text { Experiência ao mudar do ensino presencial para o ensino remoto nas disciplinas práticas } \\
\text { incluindo semiologia e semiotécnica }\end{array}$ & $1,52 \pm 0,80$ \\
\hline 23 & $\begin{array}{l}\text { Satisfação com as tecnologias utilizadas pela instituição quando foi necessário substituir } \\
\text { as aulas práticas por aulas remotas nas disciplinas práticas incluindo semiologia e } \\
\text { semiotécnica }\end{array}$ & $2,33 \pm 1,10$ \\
\hline 24 & $\begin{array}{l}\text { Preparação para executar procedimentos técnicos de semiologia e semiotécnica após as } \\
\text { aulas remotas }\end{array}$ & $1,86 \pm 1,07$ \\
\hline
\end{tabular}

Fonte: Autores.

O ensino a distância $(\mathrm{EaD})$ foi originalmente criado através de iniciativa privada e governamental, sendo estabelecido no Brasil junto com as diversas mudanças, principalmente no que se refere ao uso e mudança das tecnologias (Gomes,2013). Seu significado foi trazido de forma oficial para o Brasil com o decreto 5.622 de 19 de dezembro de 2005, sendo este revogado pelo decreto 9.057 de 25 de maio de 2017, que em seu primeiro artigo contextualiza a educação a distância como modalidade educacional, onde a mediação entre ensino e aprendizagem ocorra com a utilização de meios de tecnologia de informação e comunicação.

O ensino híbrido seria um meio termo entre o EAD e o ensino presencial, onde este modelo é uma das diversas abordagens pedagógicas de ensino que combina um misto de atividades presenciais e atividades realizadas de forma digital, fazendo-se uso das tecnologias de informação, com o objetivo de reafirmar o processo de ensino e aprendizagem para o aluno, e não mais para o professor (Sousa et al., 2017).

Sendo uma mistura de sentimentos, ações, lições, culturas e ensinamentos distintos, frutos de uma sociedade culturalmente diferente, não se limitando apenas ao uso de metodologias ativas ou a mistura entre momentos presenciais, em espaços físicos, e virtuais, fornecendo uma possibilidade de ensinar e de aprender diferenciado, com um leque de possibilidades aplicáveis (Moran,2015).

A formação dos profissionais de enfermagem pela modalidade EAD não é uma indicação dos conselhos regionais de enfermagem, já que os conhecimentos teórico-práticos e a integração desde ser profissional com a sociedade e os serviços de saúde é uma das partes mais importantes da formação deste como ser profissional, e justamente por isso, no ano de 2015, os conselhos regionais de enfermagem se posicionaram em apoio ao projeto da lei $\mathrm{n}^{\circ} 2891 / 2015$, que proíbe a formação de enfermeiros e técnicos em enfermagem por meio exclusivo do ensino EAD, projeto que ainda está em trâmite para aprovação. 
Dessa forma,observa-se que os alunos das Instituições de Ensino Superior (IES), tiveram que se adaptar às aulas teóricopráticas de forma remota, relatando que as aulas presenciais são mais eficazes em seu ensino-aprendizagem pois as aulas práticas são uma forma de colocar a teoria em campo de estágio, sendo necessária para formação profissional de qualidade, notando-se insatisfeitos ao assistir aulas práticas de forma virtual pela ocorrência de perda na qualidade da formação. A necessidade de virtualização do ensino tornou-se necessário em âmbito mundial e mesmo usando diversas ferramentas de tecnologia acabam deixando lacunas, porém mesmo perdendo esse contato físico, as discussões e a construção do conhecimento continuam acontecendo no período de distanciamento (Pissaia \& Da Costa, 2021).

\section{Conclusão}

Conclui-se portanto, que o advento da COVID-19 trouxe impactos significativos na educação e na formação do enfermeiro, pois, a enfermagem é uma profissão de assistência que lida diretamente com o ser humano em todo ciclo vital.

Nesse estudo fica claro que os conhecimentos de semiologia e semiotécnica, disciplina teórico-prática, são necessários para construção da formação dos profissionais de enfermagem, pois envolvem práticas sociais, éticas e legais que se processam pelo ensino e assistência. Além disso, o cuidado com o outro não deve acontecer de forma virtual pois é real, tangível, possui corpo e forma e, portanto, a inserção dos alunos dentro dos serviços de saúde e da população é imprescindível para a formação dos futuros enfermeiros.

Assim, entende-se que é necessária uma conciliação entre o mundo digital e a formação de profissionais na área de saúde, além da necessidade de revisão criteriosa nas diretrizes curriculares nacionais para uma formação mais sólida pautada nos princípios de ética, respeito e dignidade à pessoa, família e sociedade.

Esperamos que o presente estudo sirva de referência e reflexão para outros pesquisadores que buscam a melhoria do ensino remoto e, por meio dessa pesquisa, seja possível o surgimento de outras para contribuírem com a comunidade científica acadêmica.

\section{Referências}

Abrantes, N. N. F. D. (2012). Trabalho e estudo: uma conciliação desafiante. Campina Grande: REALIZE,. http://www.editorarealize.com.br/art igo/visualizar/485.

Barreto, A. (2014). A mulher no ensino superior: distribuição e representatividade. Cadernos do GEA, n.6, (jul./dez. 2015). FLACSO, GEA; UERJ, LPP, 2012. de Barros, A. L. B. L. (2015). Anamnese e Exame Físico-: Avaliação Diagnóstica de Enfermagem no Adulto. (2a ed.), Ed. Artmed. isbn 9788536321035.

Brasil. (2012). Ministério da Saúde 2012. Resolução n466/12 do Conselho Nacional de Saúde, http://bvsms.saude.gov.br/bvs/saudelegis/cns/2013/res0466_12_12_2012.html.

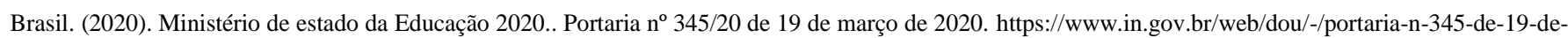
marco-de-2020-248881422? inheritRedirect=true\&redirect=\%2Fweb\%2Fguest $\% 2$ Fsearch $\% 3$ FqSearch $\% 3$ DPortaria\% $2520345 \% 2520 \mathrm{de} \% 252019 \% 2520 \mathrm{~d}$ e\%2520mar\%25C3\%25A7o\%2520de\%25202020.

Castilho, A. P., Borges, N. R. M., \& Pereira, V. T. (2014). Manual de metodologia científica. (3a ed.), Ed. Itumbiara: Iles/Ulbra, 201. https://www.ulbra.br/upload/986eb63036cdfc35003049362f114dd7.pdf

Camacho, A. C. L. F. (2020). Ensino remoto em tempos de pandemia da covid-19: novas experiências e desafios. Online braz. j. nurs. https://docs.bvsalud.org/biblioref/2021/03/1145525/6475-pt.pdf.

Cofen. (2017). Conselho Federal de Enfermagem. Relatório das Audiências Públicas Formação de Profissionais de Enfermagem na Modalidade a Distância, http://www.cofen.gov.br/relatorio-audiencias-publicas-formacao-de-profissionais-de-enfermagem-na-modalidade-ead-final_50422.html.

Ferreira, J. D. C., Santos, S. A. S., \& Tomé, M. F. (2011). Mulher e o mercado de trabalho: uma revisão sobre os percursos da mulher no mercado do trabalho. Revistas Científicas Eletrônicas da FAEF, Faculdade de Ensino Superior e Formação Integral -FAEF, 20(20), 1-7,

Fernandes, J. D., Silva, R. M. O., Cordeiro, A. L. A. O., \& Teixeira, G. A. D. S. (2021). Estágio curricular supervisionado de enfermagem em tempos de pandemia da COVID-19. Escola Anna Nery, 25. https://doi.org/10.1590/2177-9465-EAN-2021-0061 
Gil, A. C. (2008). Métodos e técnicas de pesquisa social. (6a ed.), Ed. Atlas SA.

Gomes, LF (2013). EAD no Brasil: desafios e desafios. Avaliação: Revista da Avaliação da Educação Superior (Campinas), 18, 1322.https://doi.org/10.1590/S1414-40772013000100002

Lira, A. L. B. D. C, Adamy, E. K, Teixeira, E., \& Silva, F. V. D. (2020). Educação em enfermagem: desafios e perspectivas em tempos de pandemia COVID19. Revista Brasileira de Enfermagem, 73 .https://doi.org/10.1590/0034-7167-2020-0683

Mathias, S. L., \& Sakai, C. (2013). Utilização da ferramenta google forms no processo de avaliação institucional: Estudo de caso nas Faculdades Magsul. Seminários Regionais sobre Autoavaliação Institucional e Comissões Próprias de Avaliação, 17-18. https://download.inep.gov.br/educacao_superior/avaliacao_institucional/seminarios_regionais/trabalhos_regiao/2013/centro_oeste/eixo_1/google_forms_proce sso_avaliacao_instit_estudo_caso_faculdades_mag.pdf

Moran, J. (2015). Educação híbrida: um conceito-chave para a educação, hoje. Ensino híbrido: personalização e tecnologia na educação. Porto Alegre: Penso, 27-45.http://www2.eca.usp.br/moran/wp-content/uploads/2021/01/educa\%C3\%A7\%C3\%A3o_h\%C3\%ADbrida.pdf

Melo, G. D. S. M, Tibúrcio, M. P, Freitas, C. C. S. D, Vasconcelos, Q. L. D. D. A. Q. D, Costa, I. K. F., \& Torres, GDV (2017). Semiótica e Semiologia da Enfermagem: avaliação do conhecimento dos alunos de graduação sobre procedimentos. Revista brasileira de enfermagem, 70, $249-256$.

Coren-ro. (2021). Conselho Regional de Enfermagem de Rondônia. Nota dos conselhos de enfermagem contra o EAD na enfermagem,http://www.corenro.org.br/nota-dos-conselhos-de-enfermagem-contra-o-ead-na-enfermagem_13091.html

Oliveira, S. L. D. (1997). Tratado da Metodologia Científica: projeto de pesquisa TCC, monografia (Doctoral dissertation, dissertação. São Paulo: Pioneira).

Pissaia, L. F., \& da Costa, A. E. K. (2021). Pandemia da covid-19: percepções de estudantes de enfermagem sobre o seu ensino. Oikos: Família e Sociedade em Debate, 32(1), 148-164.https://doi.org/10.31423/oikos.v32i1.11312

Porto, C. C. (2017). Semiologia Médica (10a ed.), Editora Guanabara Koogan.

Riegel, F., Martini, J. G., Bresolin, P., Mohallem, A. G. C., \& Nes, A. A. G. (2021). Desenvolvendo o pensamento crítico no ensino de Enfermagem: um desafio em tempos de pandemia de Covid-19. Escola Anna Nery, 25.https://doi.org/10.1590/2177-9465-EAN-2020-0476

Soares, A. et al. Metodologia Da Pesquisa Científica.

Segantini, J. H. (2014). O uso das tecnologias na sala de aula, como ferramenta pedagógica e seus reflexos no campo. https://www.acervodigital.ufpr.br/handle/1884/50327

Spagnol, C. A., Pereira, K. D., Castro, V. P. N. D., Figueiredo, L. G., Borges, K. K. D. S., \& Batista, L. M. (2021). Diálogos da enfermagem durante a pandemia: reflexões, desafios e perspectivas para a integração ensino-serviço. Escola Anna Nery, 25.https://doi.org/10.1590/2177-9465-EAN-2020-0498

Soares, F. A., Rocha, K. K. A., Portela, R. D. A., Silva, A. C. O., Corrêa, R. D. G. C. F., \& Oliveira, B. L. C. A. D. (2021). Cenário da educação superior à distância em saúde no Brasil: a situação da Enfermagema. Escola Anna Nery, 25.https://doi.org/10.1590/2177-9465-EAN-2020-0145

Scorsolini-Comin, F., De Melo, L. P., Rossato, L., \& Gaia, R. D. S. P. (2020). Educação a distância na formação em enfermagem: reflexões sobre a pandemia da covid-19. Revista Baiana de Enfermagem34, .

Souza, T. M., \& Chagas, A. M. (2019). Ensino híbrido: Alternativa de personalização da aprendizagem. Revista Com Censo: Estudos Educacionais do Distrito Federal, 6(1), 59-66. http://periodicos.se.df.gov.br/index.php/comcenso/article/view/587

Garcia, R. A. B., Bacarin, A. P. S., \& Leonardo, N. S. T. (2018). Acessibilidade e permanência na educação superior: percepção de estudantes com deficiência. Psicologia Escolar e Educacional, 22, 33-40. https://doi.org/10.1590/2175-3539/2018/035

Wellichan, D. D. S. P., \& da Silva Souza, C. (2017). A inclusão na prática: alunos com deficiência no ensino superior. Revista on line de Política e Gestão Educacional, 146-166. https://doi.org/10.22633/rpge.v21.n1.2017.9786 